Proceedings of the 6th Polish Symposium of Physics in Economy and Social Sciences (FENS2012), Gdańsk, Poland

\title{
Utility Functions Invariant with Respect to Some Classes of Shifts
}

\author{
J. Chudziak
}

Department of Mathematics, University of Rzeszów, T. Rejtana 16A, 35-959 Rzeszów, Poland

\begin{abstract}
A utility function $U$ is said to be invariant with respect to a family of transformations $\Gamma$ provided, for every member $\gamma$ of $\Gamma, U$ and $U \circ \gamma$ represent the same preference relation over lotteries. An invariance with respect to a wide class of transformations can be reduced to an invariance with respect to the shift transformations. We give a complete answer to the following question: given a nonempty set $T$ of shifts determine all utility functions invariant with respect to the shift transformations by every element of $T$. As a consequence of our results we obtain the forms of utility functions invariant with respect to the families of commuting transformations.
\end{abstract}

DOI: $10.12693 /$ APhysPolA.123.508

PACS: 89.65.Gh

\section{Introduction}

Let $X \neq \emptyset$. A function $p: X \rightarrow[0,1]$ is said to be a simple probability distribution (or a lottery) on $X$ provided the $\operatorname{set} \operatorname{supp}(p):=\{x \in X \mid p(x)>0\}$ is finite and $\sum_{x \in \operatorname{supp}(p)} p(x)=1$. A family of all lotteries on $X$ will be denoted by $\Delta(X)$. According to the classical result of von Neumann and Morgenstern, every preference relation $\succeq$ on $\Delta(X)$ satisfying some additional assumptions can be represented by a utility function, that is there exists a function $U: \Delta(X) \rightarrow \mathbb{R}$ such that, for every $p, q \in \Delta(\mathbb{R})$, we have

$$
p \succeq q \Longleftrightarrow U(p) \geq U(q) \text {. }
$$

Furthermore, every such a function possesses the so-called Bernoulli utility function, that is a function $u$ : $X \rightarrow \mathbb{R}$ such that

$$
U(p)=\sum_{x \in \operatorname{supp}(p)} p(x) u(x) \quad \text { for } \quad p \in \Delta(X) .
$$

We will also use the fact that two utility functions $U$ and $V$ having the Bernoulli utility functions $u$ and $v$, respectively, represent the same preference relation over lotteries if and only if there exist $a \in(0, \infty)$ and $b \in \mathbb{R}$ such that

$$
v(x)=a u(x)+b \quad \text { for } \quad x \in X .
$$

An important problem in a decision analysis under risk is to determine a form of a utility function representing a decision maker's preference relation over lotteries. One of the approaches to this problem is based on a notion of invariance studied for the first time by Pfanzagl [1]. Given a nonempty set $T$, a utility function $U: \Delta(X) \rightarrow \mathbb{R}$ is said to be invariant with respect to a family of transformations $\Gamma=\left\{\gamma_{t}: X \rightarrow X \mid t \in T\right\}$ provided, for every $t \in T, U$ and $U \circ \gamma_{t}$ represent the same preference relation over lotteries.

Let us illustrate this notion by the following example. Consider a family of bonds on some market. Suppose that the profits from the bonds are related to the inflation rate. Furthermore, assume that there are some predictions concerning the inflation rate and so the possible levels of profits from every bond in a fixed period (e.g. a year). More precisely, suppose that for every bond $\mathbb{B}$ there exist a finite set of positive outcomes $\left\{x_{1}, \ldots, x_{n}\right\}$ and a finite set of nonzero probabilities $\left\{p_{1}, \ldots, p_{n}\right\}$ $\left(p_{1}, \ldots, p_{n} \in(0,1], \sum_{i=1}^{n} p_{i}=1\right)$ such that at the end of the period a profit from the bond will be equal to $x_{i}$ with a probability $p_{i}, i \in\{1, \ldots, n\}$. In this way every bond $\mathbb{B}$ can be treated as a lottery which pays $x_{i}$ with probability $p_{i}$, i.e. $\mathbb{B}=\left\langle x_{1}, p_{1} ; \ldots, x_{n}, p_{n}\right\rangle$. Assume that the investor's preference relation over the bonds (lotteries) is represented by a von Neumann-Morgenstern utility function and consider two situations.

1. A discount is offered to the investor, that is he can pay less, say $t$ euro less, for every bond. This means that he can consider a bond $\mathbb{B}=\left\langle x_{1}, p_{1} ; \ldots, x_{n}, p_{n}\right\rangle$ as the following one $\mathbb{B}_{+t}=\left\langle x_{1}+t, p_{1} ; \ldots, x_{n}+t, p_{n}\right\rangle$. If this offer does not change the investor's preference relation then his utility function is said to be invariant with respect to the shift by $t$.

2. A new capital gains tax, say $\beta \%$, is announced. In such a case every bond $\mathbb{B}=\left\langle x_{1}, p_{1} ; \ldots, x_{n}, p_{n}\right\rangle$ can be considered as $\mathbb{B}_{\delta}=\left\langle\delta x_{1}, p_{1} ; \ldots, \delta x_{n}, p_{n}\right\rangle$, where $\delta:=$ $1-\beta$. If this fact does not change the investor's preference relation, then his utility function is said to be invariant with respect to the scale transformation by $\delta$.

Recently, Abbas [2] noted that an invariance with respect to a wide class of transformations can be reduced to an invariance with respect to the shift transformations. More precisely (see Theorem 1 ), if $I \subseteq \mathbb{R}$ is an open interval, $T \neq \emptyset, G: I \rightarrow \mathbb{R}$ is a continuous bijection and $H: T \rightarrow \mathbb{R}$, then an invariance with respect to a family of transformations $\Gamma=\left\{\gamma_{t}: I \rightarrow I \mid t \in T\right\}$, where

$$
\gamma_{t}(x)=G^{-1}[G(x)+H(t)] \text { for } \quad x \in I, t \in T
$$

can be reduced to an invariance with respect to the family of shifts by every element of $H(T)$. Let us note that (1) defines a wide family of transformations including, as the particular cases, the shift transformations $(I=\mathbb{R}$, $G(x)=x$ for $x \in \mathbb{R}, H(t)=t$ for $t \in T)$ and the scale transformations $(I=(0, \infty), G(x)=\ln x$ for $x \in(0, \infty)$, $T \subseteq(0, \infty), H(t)=\ln t$ for $t \in T)$. 
Furthermore, every two members of $\Gamma$ commute, that is $\gamma_{t} \circ \gamma_{s}=\gamma_{s} \circ \gamma_{t}$ for $s, t \in T$. It is remarkable that under some additional natural assumptions, every family of commuting mappings $\gamma_{t}: I \rightarrow I$ consists of maps of the form (10) with some bijection $G: I \rightarrow \mathbb{R}$ and $H: T \rightarrow \mathbb{R}$. More details concerning this problem can be found in [3] and [4].

Invariant multiattribute utility functions have been studied in [2 and 5-7]. As we already mentioned, Abbas [2] proved that a crucial role in an invariance problem is played by utility functions invariant with respect to the shift transformations. Furthermore, in [5] and [8] it is has been shown that the utility functions invariant with respect to a single shift transformations (or even with respect to the shift transformations by infinitely many parameters) may depend on an arbitrary periodic function. Since such functions are not useful for applications, it is natural to ask when such "strage" utility functions can be eliminated.

In this paper we give a complete answer to the following question: given a nonempty set $T$ of shifts determines all utility functions invariant with respect to the shift transformations by every element of $T$. As a consequence of our results we obtain the forms of utility functions invariant with respect to the families of commuting transformations. In this way we generalize the results in [8].

In the next section we present the main results of the paper and two examples, which show the possible applications of the results. The proofs are included in the third section.

\section{Results}

The first result of this section concerns the connections between an invariance property and a behavior of a Bernoulli utility function. The second one is a reduction theorem (it is closely related to [8, Proposition 1, p. 19], but for a convienience of the reader we include its short proof).

Proposition 1. Assume that $X \neq \emptyset$ and $U: \Delta(X) \rightarrow$ $\mathbb{R}$ is a utility function representing a decision maker's preference relation over $\Delta(X)$. Let $u: X \rightarrow \mathbb{R}$ be a Bernoulli utility function related to $U$. Then $U$ is invariant with respect to a family of transformations $\left\{\gamma_{t}\right.$ : $X \rightarrow X \mid t \in T\}$, where $T \neq \emptyset$, if and only if there exist functions $k: T \rightarrow(0, \infty)$ and $l: T \rightarrow \mathbb{R}$ such that

$$
u\left(\gamma_{t}(x)\right)=k(t) u(x)+l(t) \quad \text { for } \quad x \in \mathbb{R}, t \in T .
$$

Theorem 1. Let $I \subseteq \mathbb{R}$ be an open interval and let $T$ be a nonempty subset of $\mathbb{R}$. A utility function $U: \Delta(I) \rightarrow$ $\mathbb{R}$ having a Bernoulli utility function $u: I \rightarrow \mathbb{R}$ is invariant with respect to a family $\Gamma=\left\{\gamma_{t}: I \rightarrow I \mid t \in T\right\}$ of transformations of the form (1) with some continuous bijection $G: I \rightarrow \mathbb{R}$ and $H: T \rightarrow \mathbb{R}$ if and only if a utility function $\tilde{U}: \Delta(\mathbb{R}) \rightarrow \mathbb{R}$ with a Bernoulli utility function $\tilde{u}:=u \circ G^{-1}: \mathbb{R} \rightarrow \mathbb{R}$ is invariant with respect to the shift transformations by every element of $H(T)$.

The next theorem is the main result of the paper.
Theorem 2. Let $T$ be a nonempty subset of $\mathbb{R} \backslash\{0\}$ and let $[T]$ denote a subgroup of the additive group of real numbers generated by T. A utility function $U: \Delta(\mathbb{R}) \rightarrow$ $\mathbb{R}$ having a continuous Bernoulli utility function $u: \mathbb{R} \rightarrow$ $\mathbb{R}$ is invariant with respect to the shift transformations by every element of $T$ if and only if one of the following conditions holds:

(i) $[T]=d \mathbb{Z}:=\{d n \mid n \in \mathbb{Z}\}$ for some $d \in \mathbb{R} \backslash\{0\}$ and there exists a continuous d-periodic function $p: \mathbb{R} \rightarrow \mathbb{R}$ such that either

$$
u(x)=c x+p(x) \quad \text { for } \quad x \in \mathbb{R}
$$

with some $c \in \mathbb{R}$, or

$$
u(x)=a^{x} p(x)+b \quad \text { for } \quad x \in \mathbb{R}
$$

with some $a \in(0, \infty) \backslash\{1\}$ and $b \in \mathbb{R}$;

(ii) $[T]$ is a dense subset of $\mathbb{R}$ and either

$$
u(x)=c x+b \quad \text { for } \quad x \in \mathbb{R}
$$

with some $b, c \in \mathbb{R}$, or

$$
u(x)=c a^{x}+b \text { for } x \in \mathbb{R}
$$

with some $a \in(0, \infty) \backslash\{1\}$ and $b, c \in \mathbb{R}$.

From Theorems 1 and 2 it follows the result concerning utility functions invariant with respect to the family $\Gamma$ of transformations of the form (1).

Theorem 3. Let $I \subseteq \mathbb{R}$ be an open interval and $T \neq \emptyset$. Assume that $G: I \rightarrow \mathbb{R}$ is a continuous bijection and $H: T \rightarrow \mathbb{R}$. Utility function $U: \Delta(I) \rightarrow \mathbb{R}$ having a continuous Bernoulli utility function $u: I \rightarrow \mathbb{R}$ is invariant with respect to the family $\Gamma=\left\{\gamma_{t}: I \rightarrow I \mid t \in T\right\}$ of transformations of the form (1) if and only if one of the following conditions holds:

(i) $[H(T)]=d \mathbb{Z}$ for some $d \in \mathbb{R} \backslash\{0\}$ and there exists a d-periodic continuous function $p: \mathbb{R} \rightarrow \mathbb{R}$ such that either

$$
u(x)=c G(x)+p(G(x)) \quad \text { for } \quad x \in I
$$

with some $c \in \mathbb{R}$, or

$$
u(x)=a^{G(x)} p(G(x))+b \quad \text { for } \quad x \in I
$$

with some $a \in(0, \infty) \backslash\{1\}$ and $b \in \mathbb{R}$;

$($ ii $)[H(T)]$ is a dense subset of $\mathbb{R}$ and either

$$
u(x)=c G(x)+b \quad \text { for } \quad x \in I,
$$

with some $b, c \in \mathbb{R}$, or

$$
u(x)=c a^{G(x)}+b \quad \text { for } \quad x \in I
$$

with some $a \in(0, \infty) \backslash\{1\}$ and $b, c \in \mathbb{R}$.

We conclude this section with two examples presenting the possible applications of Theorems 2 and 3 (cf. [2, Example 2, p. 79] and [8, pp. 20-22 and 28-29])

Example 1. Consider the example described in Introduction. If the investor gets a discount $t$ euro $(t>0)$ for every bond and this offer does not change his preference relation over bonds then his utility function is invariant with respect to a shift transformation by $t$. Thus $T=\{t\}$ and so $[T]=t \mathbb{Z}$. Therefore, if the investor's Bernoulli utility function $u: \mathbb{R} \rightarrow \mathbb{R}$ is continuous, then according to Theorem 2 there exists a continuous $t$-periodic function $p: \mathbb{R} \rightarrow \mathbb{R}$ such that $u$ is either of the form (3) with some $c \in \mathbb{R}$, or (4) with some $a \in(0, \infty) \backslash\{1\}$ and $b \in \mathbb{R}$. 
However, if any discount from some interval $T$ does not change the investor's preference relation then $[T]=\mathbb{R}$ and so, applying Theorem 2, we obtain that $u$ is either of the form (5) with some $b, c \in \mathbb{R}$, or (6) with some $a \in(0, \infty) \backslash\{1\}$ and $b, c \in \mathbb{R}$.

Example 2. Let us continue the previous example, but now assume that the second possibility described in Introduction occurs. If a new capital gains $\operatorname{tax} \beta \%$ does not change the investor's preference relation over bonds, then his utility function is invariant with respect to a scale transformation $\gamma_{\delta}:(0, \infty) \rightarrow(0, \infty), \gamma_{\delta}(x)=\delta x$ for $x \in(0, \infty)$, where $\delta:=1-\beta$. Let us note that $\gamma_{\delta}$ is of the form (1) with $I=(0, \infty), T=\{\delta\}, G(x)=$ $\ln x$ for $x \in(0, \infty)$ and $H(\delta)=\ln \delta$. Since $[H(T)]=$ $(\ln \delta) \mathbb{Z}$ and $a^{\ln x}=x^{\ln a}$ for $a, x \in(0, \infty)$, if the investor's Bernoulli utility function $u:(0, \infty) \rightarrow \mathbb{R}$ is continuous, then according to Theorem 3 there exists a continuous $(\ln \delta)$-periodic function $p: \mathbb{R} \rightarrow \mathbb{R}$ such that either

$$
u(x)=c \ln x+p(\ln x) \quad \text { for } \quad x \in(0, \infty)
$$

with some $c \in \mathbb{R}$, or

$$
u(x)=x^{s} p(\ln x)+b \quad \text { for } \quad x \in(0, \infty)
$$

with some $s \in \mathbb{R} \backslash\{0\}$ and $b \in \mathbb{R}$.

If any capital gains tax from some interval $T$ does not change the investor's preference relation, then $[H(T)]=$ $\mathbb{R}$, so applying Theorem 3 , we obtain that either

$$
u(x)=c \ln x+b \quad \text { for } \quad x \in(0, \infty)
$$

with some $b, c \in \mathbb{R}$, or

$$
u(x)=c x^{s}+b \quad \text { for } \quad x \in(0, \infty)
$$

with some $s \in \mathbb{R} \backslash\{0\}$ and $b, c \in \mathbb{R}$.

\section{Proofs}

Proof of Proposition 1: Assume that $U$ is invariant with respect to the family of transformations $\left\{\gamma_{t}: X \rightarrow\right.$ $X \mid t \in T\}$. Then, for every $t \in T$, a utility function $U_{t}: \Delta(X) \rightarrow \mathbb{R}$ given by

$$
U_{t}(p)=U\left(p_{\gamma_{t}}\right) \text { for } p=\left\langle x_{1}, p_{1} ; \ldots, x_{n}, p_{n}\right\rangle \in \Delta(X),
$$

where $p_{\gamma_{t}}=\left\langle\gamma_{t}\left(x_{1}\right), p_{1} ; \ldots, \gamma_{t}\left(x_{n}\right), p_{n}\right\rangle$, represents the same preference relation as $U$. Therefore if, for every $t \in T, u_{t}$ denotes a Bernoulli utility function related to $U_{t}$, then we have

$$
u_{t}(x)=k(t) u(x)+l(t) \quad \text { for } \quad x \in X, t \in T
$$

with some $k(t) \in(0, \infty)$ and $l(t) \in \mathbb{R}$. Moreover, let us note that

$$
u_{t}(x)=u\left(\gamma_{t}(x)\right) \quad \text { for } \quad x \in X, t \in T .
$$

Then (2) follows from (7) and (8).

Conversely, if (2) holds then, for every $t \in T$, a utility function $U_{t}$ with a Bernoulli utility function $u_{t}$ given by (8) represents the same preference relation as $U$. Thus $U$ is invariant with respect to the family $\left\{\gamma_{t}: X \rightarrow X \mid\right.$ $t \in T\}$.

Proof of Theorem 1: Assume that a utility function $U: I \rightarrow \mathbb{R}$ is invariant with respect to the family $\Gamma$. The case where $u$ is constant is trivial. So, assume that $u$ is nonconstant. Then $\tilde{u}$ is also nonconstant. Moreover, according to Proposition 1, there exist functions $k: T \rightarrow$ $(0, \infty)$ and $l: T \rightarrow \mathbb{R}$ such that

$$
\begin{aligned}
& u\left(G^{-1}(G(x)+H(t))\right)=k(t) u(x)+l(t) \\
& \quad \text { for } \quad x \in I, t \in T .
\end{aligned}
$$

Hence

$$
\begin{aligned}
& \tilde{u}(G(x)+H(t))=k(t) \tilde{u}(G(x))+l(t) \\
& \quad \text { for } \quad x \in I, t \in T .
\end{aligned}
$$

Since $G$ maps $I$ onto $\mathbb{R}$ this implies that

$$
\tilde{u}(x+H(t))=k(t) \tilde{u}(x)+l(t) \quad \text { for } \quad x \in \mathbb{R}, t \in T .
$$

Since $\tilde{u}$ is nonconstant, taking $x_{1}, x_{2} \in \mathbb{R}$ with $\tilde{u}\left(x_{1}\right) \neq$ $\tilde{u}\left(x_{2}\right)$, from (9) we derive that $\tilde{u}\left(x_{i}+H(t)\right)=k(t) \tilde{u}\left(x_{i}\right)+$ $l(t)$ for $t \in T$ and $i \in\{1,2\}$. Hence, after straightforward calculations, we get $k(t)=K(H(t))$ for $t \in T$ and $l(t)=$ $L(H(t))$ for $t \in T$, where $K: H(T) \rightarrow(0, \infty)$ and $L:$ $H(T) \rightarrow \mathbb{R}$ are given by

$$
K(s)=\frac{\tilde{u}\left(x_{1}+s\right)-\tilde{u}\left(x_{2}+s\right)}{\tilde{u}\left(x_{1}\right)-\tilde{u}\left(x_{2}\right)} \quad \text { for } \quad s \in H(T)
$$

and

$$
\begin{aligned}
& L(s)=\frac{\tilde{u}\left(x_{1}\right) \tilde{u}\left(x_{2}+s\right)-\tilde{u}\left(x_{2}\right) \tilde{u}\left(x_{1}+s\right)}{\tilde{u}\left(x_{1}\right)-\tilde{u}\left(x_{2}\right)} \\
& \quad \text { for } \quad s \in H(T),
\end{aligned}
$$

respectively. Thus, taking into account (9), we get

$$
\begin{aligned}
& \tilde{u}(x+H(t))=K(H(t)) \tilde{u}(x)+L(H(t)) \\
& \quad \text { for } \quad x \in \mathbb{R}, t \in T .
\end{aligned}
$$

Hence

$$
\tilde{u}(x+s)=K(s) \tilde{u}(x)+L(s) \quad \text { for } \quad x \in \mathbb{R}, s \in H(T) .
$$

Therefore, applying Proposition 1 , we conclude that $\tilde{U}$ is invariant with respect to the shifts transformations by every element of $H(T)$.

Now, assume that $\tilde{U}$ is invariant with respect to the shifts transformations by every element of $H(T)$. Then, according to Proposition 1, there exist functions $\tilde{k}$ : $H(T) \rightarrow(0, \infty)$ and $\tilde{l}: H(T) \rightarrow \mathbb{R}$ such that

$$
\tilde{u}(x+s)=\tilde{k}(s) \tilde{u}(x)+\tilde{l}(s) \quad \text { for } \quad x \in \mathbb{R}, s \in H(T) .
$$

Hence

$$
\begin{aligned}
& \tilde{u}(G(x)+H(t))=\tilde{k}(H(t)) \tilde{u}(G(x))+\tilde{l}(H(t)) \\
& \quad \text { for } \quad x \in I, t \in T
\end{aligned}
$$

and so

$$
\begin{aligned}
& u\left(G^{-1}(G(x)+H(t))\right)=(\tilde{k} \circ H)(t) u(x)+(\tilde{l} \circ H)(t) \\
& \quad \text { for } \quad x \in I, t \in T .
\end{aligned}
$$

Thus, applying Proposition 1 again, we obtain that a utility function $U$ is invariant with respect to the family $\Gamma$.

Proof of Theorem 2: Assume that (i) holds and fix $x \in \mathbb{R}$ and $t \in T$. Then $t=d n$ with some $n \in \mathbb{Z}$. So, in the case where $u$ is of the form (3), we have

$$
u(x+t)=u(x+d n)=c(x+d n)+p(x+d n)
$$


$=c x+p(x)+c d n=u(x)+c t$.

In the case where $u$ is of the form (4), we obtain

$$
\begin{gathered}
u(x+t)=u(x+d n)=a^{x+d n} p(x+d n)+b \\
=a^{d n} a^{x} p(x)+b=a^{t} u(x)+b\left(1-a^{t}\right) .
\end{gathered}
$$

Now, assume that (ii) holds. Then, in the case where $u$ is of the form (5), we get

$$
\begin{aligned}
& u(x+t)=c(x+t)+b=c x+b+c t=u(x)+c t \\
& \quad \text { for } \quad x \in \mathbb{R}, t \in T
\end{aligned}
$$

and, in the case of (6), we have

$$
\begin{aligned}
& u(x+t)=c a^{x+t}+b=a^{t}\left(c a^{x}+b\right)+b\left(1-a^{t}\right) \\
& =a^{t} u(x)+b\left(1-a^{t}\right) \quad \text { for } \quad x \in \mathbb{R}, t \in T .
\end{aligned}
$$

Therefore, applying Proposition 1, we obtain that $U$ is invariant with respect to the shift transformations by every element of $T$.

Now, assume that $U$ is invariant with respect to the shift transformations by every element of $T$. Then, according to Proposition 1, there exist functions $k: T \rightarrow$ $(0, \infty)$ and $l: T \rightarrow \mathbb{R}$ such that

$$
u(x+t)=k(t) u(x)+l(t) \quad \text { for } \quad x \in \mathbb{R}, t \in T .
$$

The case where $u$ is constant is trivial. So, assume that $u$ is nonconstant. The remaining part of the proof is divided into three steps.

Step 1. We show that for every $t \in T$ one of the following two possibilities holds:

(a) $k(1)=1$ and there is a $t$-periodic function $p_{t}: \mathbb{R} \rightarrow$ $\mathbb{R}$ such that

$$
u(x)=\frac{l(t)}{t} x+p_{t}(x) \quad \text { for } \quad x \in \mathbb{R} ;
$$

(b) $k(t) \neq 1$ and there is a $t$-periodic function $p_{t}: \mathbb{R} \rightarrow$ $\mathbb{R}$ such that

$$
u(x)=k(t)^{\frac{x}{t}} p_{t}(x)+\frac{l(t)}{1-k(t)} \quad \text { for } \quad x \in \mathbb{R} .
$$

To this end, fix a $t \in T$. If $k(t)=1$ then, in view of (10), a function $F:=\mathrm{e}^{u}$ satisfies the equation $F(x+t)=$ $\mathrm{e}^{l(t)} F(x)$ for $x \in \mathbb{R}$. Therefore, a function $\tilde{p}_{t}: \mathbb{R} \rightarrow \mathbb{R}$ of the form $\tilde{p}_{t}(x)=\exp \left(-\frac{l(t) x}{t}\right) F(x)$ for $x \in \mathbb{R}$, satisfies

$$
\begin{aligned}
& \tilde{p}_{t}(x+t)=\exp \left(-\frac{l(t)(x+t)}{t}\right) F(x+t) \\
& =\exp \left(-\frac{l(t) x}{t}\right) \mathrm{e}^{-l(t)} \mathrm{e}^{l(t)} F(x)=\tilde{p}_{t}(x) \\
& \quad \text { for } \quad x \in \mathbb{R} .
\end{aligned}
$$

Thus, $\tilde{p}_{t}$ is a $t$-periodic function and $F(x)=$ $\exp \left(\frac{l(t) x}{t}\right) \tilde{p}_{t}(x)$ for $x \in \mathbb{R}$. Hence $u$ is of the form (11) with $p_{t}:=\ln \tilde{p}_{t}$. So, $(a)$ holds.

Now, assume that $k(t) \neq 1$. Then taking a function $\tilde{u}:=u-\frac{l(t)}{1-k(t)}$, in view of (10), for every $x \in \mathbb{R}$, we obtain

$$
\tilde{u}(x+t)=u(x+t)-\frac{l(t)}{1-k(t)}=k(t) u(x)+l(t)
$$

$$
-\frac{l(t)}{1-k(t)}=k(t)\left(u-\frac{l(t)}{1-k(t)}\right)=k(t) \tilde{u}(x) .
$$

Hence, a function $p_{t}: \mathbb{R} \rightarrow \mathbb{R}$ of the form $p_{t}(x)=$ $k(t)^{-\frac{x}{t}} \tilde{u}(x)$ for $x \in \mathbb{R}$, satisfies

$$
\begin{aligned}
& p_{t}(x+t)=k(t)^{-\frac{x+t}{t}} \tilde{u}(x+t)=k(t)^{-\frac{x}{t}-1} k(t) \tilde{u}(x) \\
& \quad=p_{t}(x) \text { for } \quad x \in \mathbb{R} .
\end{aligned}
$$

Therefore, $p_{t}$ is $t$-periodic. Furthermore, we have $\tilde{u}(x)=$ $k(t)^{\frac{x}{t}} p_{t}(x)$ for $x \in \mathbb{R}$, which implies (12). Thus, (b) holds.

Step 2. We show that either $(a)$ holds for every $t \in T$, or (b) holds for every $t \in T$. For the proof by contradiction, suppose that $(a)$ holds for some $t_{1} \in T$ and $(b)$ holds for some $t_{2} \in T$. Then, according to Step 1, there exist $t_{i}$-periodic functions $p_{i}: \mathbb{R} \rightarrow \mathbb{R}$ for $i \in\{1,2\}$ such that

$$
u(x)=\frac{l\left(t_{1}\right)}{t_{1}} x+p_{1}(x) \quad \text { for } \quad x \in \mathbb{R}
$$

and

$$
u(x)=k\left(t_{2}\right)^{\frac{x}{t_{2}}} p_{2}(x)+\frac{l\left(t_{2}\right)}{1-k\left(t_{2}\right)} \quad \text { for } \quad x \in \mathbb{R} .
$$

Since $u$ is continuous, from (13) and (14) it follows that so are $p_{1}$ and $p_{2}$. Thus $p_{1}$ and $p_{2}$, being periodic, are bounded. Furthermore, as $u$ is nonconstant, $p_{2}$ is not identically 0 . Therefore (13) implies that

$$
\lim _{x \rightarrow-\infty} \frac{u(x)}{x}=\lim _{x \rightarrow \infty} \frac{u(x)}{x}=\frac{l\left(t_{1}\right)}{t_{1}} .
$$

So, in view of (14), we get

$$
\lim _{x \rightarrow-\infty} \frac{k\left(t_{2}\right)^{\frac{x}{t_{2}}}}{x} p_{2}(x)=\lim _{x \rightarrow \infty} \frac{k\left(t_{2}\right)^{\frac{x}{t_{2}}}}{x} p_{2}(x)=\frac{l\left(t_{1}\right)}{t_{1}} .
$$

On the other hand, taking $x_{0} \in \mathbb{R}$ with $p_{2}\left(x_{0}\right) \neq 0$ and using the fact that $p_{2}$ is $t_{2}$-periodic, we have

$$
\begin{aligned}
& \lim _{n \rightarrow \infty}\left|\frac{k\left(t_{2}\right)^{\frac{x_{0}+n t_{2}}{t_{2}}}}{x_{0}+n t_{2}} p_{2}\left(x_{0}+n t_{2}\right)\right| \\
& \quad=\left|p_{2}\left(x_{0}\right) k\left(t_{2}\right)^{\frac{x_{0}}{t_{2}}}\right| \lim _{n \rightarrow \infty}\left|\frac{k\left(t_{2}\right)^{n}}{x_{0}+n t_{2}}\right|=\infty
\end{aligned}
$$

in the case where $k\left(t_{2}\right)>1$; and

$$
\begin{aligned}
& \lim _{n \rightarrow \infty}\left|\frac{k\left(t_{2}\right)^{\frac{x_{0}-n t_{2}}{t_{2}}}}{x_{0}-n t_{2}} p_{2}\left(x_{0}-n t_{2}\right)\right| \\
& \quad=\left|p_{2}\left(x_{0}\right) k\left(t_{2}\right)^{\frac{x_{0}}{t_{2}}}\right| \lim _{n \rightarrow \infty}\left|\frac{k\left(t_{2}\right)^{-n}}{x_{0}-n t_{2}}\right|=\infty
\end{aligned}
$$

in the case where $k\left(t_{2}\right)<1$. This yields a contradiction.

Step 3. First consider the case where $(a)$ holds for every $t \in T$. Clearly, we have $k=1$. Fix a $t_{0} \in T$. Then there exists a $t_{0}$-periodic function $p_{t_{0}}: \mathbb{R} \rightarrow \mathbb{R}$ such that

$$
u(x)=\frac{l\left(t_{0}\right)}{t_{0}} x+p_{t_{0}}(x) \quad \text { for } \quad x \in \mathbb{R} .
$$

Moreover, for every $t \in T$, there exists a $t$-periodic function $p_{t}: \mathbb{R} \rightarrow \mathbb{R}$ such that (11) holds. Hence, in view of (15), we obtain 


$$
\left(\frac{l\left(t_{0}\right)}{t_{0}}-\frac{l(t)}{t}\right) x=p_{t}(x)-p_{t_{0}}(x) \quad \text { for } \quad x \in \mathbb{R} .
$$

Since $p_{t}$ and $p_{t_{0}}$ are continuous periodic functions, they are bounded. Therefore, a right hand side of (16) is bounded. Hence, the left hand side of (16) is bounded as well. Thus $\frac{l\left(t_{0}\right)}{t_{0}}=\frac{l(t)}{t}$ and so $p_{t}=p_{t_{0}}$. Consequently, taking $c:=\frac{l\left(t_{0}\right)}{t_{0}}$ and $p:=p_{t_{0}}$, in view of $(15)$, we get that $p$ is continuous, (3) holds and

$$
p(x+t)=p(x) \quad \text { for } \quad x \in \mathbb{R}, t \in T .
$$

Next, consider the case where $(b)$ holds for every $t \in T$. Then, taking a $t_{0} \in T$, we get that there exists a $t_{0^{-}}$ -periodic function $p_{t_{0}}: \mathbb{R} \rightarrow \mathbb{R}$ such that

$$
u(x)=a^{x} p_{t_{0}}(x)+b \text { for } x \in \mathbb{R},
$$

where $a:=k\left(t_{0}\right)^{\frac{1}{t_{0}}} \in(0, \infty) \backslash\{1\}$ and $b:=\frac{l\left(t_{0}\right)}{1-k\left(t_{0}\right)}$. Moreover, putting into (10) $u$ of the form (18), we obtain

$$
\begin{aligned}
& a^{x}\left[a^{t} p_{t_{0}}(x+t)-k(t) p_{t_{0}}(x)\right]=l(t)-b[1-k(t)] \\
& \quad \text { for } \quad x \in \mathbb{R}, t \in T .
\end{aligned}
$$

Hence

$$
\begin{aligned}
a^{t_{0}} & {[l(t)-b(1-k(t))] } \\
& =a^{x+t_{0}}\left[a^{t} p_{t_{0}}(x+t)-k(t) p_{t_{0}}(x)\right] \\
& =a^{x+t_{0}}\left[a^{t} p_{t_{0}}\left(x+t_{0}+t\right)-k(t) p_{t_{0}}\left(x+t_{0}\right)\right] \\
& =l(t)-b(1-k(t)) \quad \text { for } \quad x \in \mathbb{R}, t \in T .
\end{aligned}
$$

Since $a \neq 1$ and $t_{0} \in T \subset \mathbb{R} \backslash\{0\}$, this implies that $l(t)=$ $b(1-k(t))$ for $t \in T$. Therefore, taking into account (12), we obtain that for every $t \in T$ there exists a $t$-periodic function $p_{t}: \mathbb{R} \rightarrow \mathbb{R}$ such that $u(x)=k(t)^{\frac{x}{t}} p_{t}(x)+b$ for $x \in \mathbb{R}$. As $u$ is nonconstant, we have $p_{t}\left(x_{0}\right) \neq 0$ for some $x_{0} \in \mathbb{R}$. Moreover, taking into account (18), we get

$$
a^{x} p_{t_{0}}(x)=k(t)^{\frac{x}{t}} p_{t}(x) \quad \text { for } \quad x \in \mathbb{R}, t \in T
$$

and so

$$
\left(\frac{k(t)^{\frac{1}{t}}}{a}\right)^{x} p_{t}(x)=p_{t_{0}}(x) \quad \text { for } \quad x \in \mathbb{R}, t \in T .
$$

Hence

$$
\begin{aligned}
& \left(\frac{k(t)^{\frac{1}{t}}}{a}\right)^{x_{0}+n t}=\frac{p_{t_{0}}\left(x_{0}+n t\right)}{p_{t}\left(x_{0}+n t\right)}=\frac{p_{t_{0}}\left(x_{0}+n t\right)}{p_{t}\left(x_{0}\right)} \\
& \quad \text { for } n \in \mathbb{Z}, t \in T .
\end{aligned}
$$

Since $p_{t_{0}}$, being a continuous periodic function, is bounded, this implies that $\frac{k(t)^{\frac{1}{t}}}{a}=1$ for $t \in T$ and so, by (19), we get $p_{t_{0}}=p_{t}$ for $t \in T$. Therefore, taking $p:=p_{t_{0}}$, in view of (18), we obtain that $p$ is continuous, $u$ is of the form (4) and (17) holds.

Finally, from (17) it follows that $p(x+t)=p(x)$ for $x \in \mathbb{R}, t \in[T]$. Moreover $[T]$, being a subgroup of the additive group of real numbers, either is cyclic or dense. In the first case we get (i). In the latter one $p$ is constant, so in view of (3) and (4), we obtain (ii).

\section{References}

[1] J. Pfanzagl, Naval Res. Logist. Quarterly 6, 283 (1959).

[2] A.E. Abbas, Theory Decision 68, 69 (2010).

[3] J. Aczél, A.D. Wallace, Colloq. Math. 17, 29 (1967).

[4] M. Hosszú, Publ. Math. Debrecen 9, 105 (1962).

[5] A.E. Abbas, J. Aczél, Decision Anal. 7, 215 (2010).

[6] A.E. Abbas, J. Aczél, J. Chudziak, Result. Math. 54, 1 (2009).

[7] J. Chudziak, Acta Phys. Pol. A 117, 673 (2010).

[8] A.E. Abbas, Decision Analysis 4, 17 (2007). 